\title{
Iliofemoral Venous Thrombosis Mainly Related to Iliofemoral Venous Obstruction by External Tumor Compression in Cancer Patients
}

\author{
Tzu-Yao Liao ${ }^{a}$ Hui-Ching Hsu ${ }^{b}$ Min-Sheng Wen ${ }^{c}$ Yu-Hsiang Juan ${ }^{d}$ \\ Yu-Hsin Hung ${ }^{\text {a Chuang-Chi Liaw }}{ }^{a}$ \\ ${ }^{a}$ Division of Hemato-Oncology, Department of Internal Medicine, Chang Gung Memorial \\ Hospital and College of Medicine, Chang Gung University, Taoyuan, Taiwan; ${ }^{b}$ Division of \\ Chinese Acupuncture and Traumatology, Department of Traditional Chinese Medicine, \\ Chang Gung Memorial Hospital and Chang Gung University, Taoyuan, Taiwan; 'Division of \\ Cardiology, Department of Internal Medicine, Chang Gung Memorial Hospital and College \\ of Medicine, Chang Gung University, Taoyuan, Taiwan; ${ }^{\mathrm{d}}$ Department of Medical Imaging \\ and Intervention, Chang Gung Memorial Hospital and College of Medicine, Chang Gung \\ University, Taoyuan, Taiwan
}

\section{Keywords}

Iliofemoral obstruction · Thrombosis · Deep vein thrombosis · D-dimer · Computed tomography · Duplex ultrasound

\section{Abstract}

Background: To study iliofemoral venous thrombosis related to iliofemoral venous obstruction in cancer patients. Methods: In this case series study, 829 cancer patients were surveyed for iliofemoral obstruction/thrombosis within 10 years. The criteria for inclusion were: (1) 


\section{Case Reports in Oncology}

presence of unilateral lower-extremity swelling; (2) computed tomography (CT) scans showing a tumor with external compression of the iliac or femoral vein, and (3) duplex ultrasound scans showing venous thrombosis or venous flow insufficiency over a femoral vein or saphenous vein. Results: Sixty-three patients (8\%) developed an iliofemoral venous obstruction. The presence of iliofemoral venous thrombosis was detected in 21 of these patients (33\%). The rate of iliofemoral venous thrombosis was significantly higher in patients with an invasion of the inguinal region, D-dimer levels $>3,000 \mathrm{ng} / \mathrm{ml}$, gastrointestinal cancer, or invasion of the inguinal lymph nodes. However, none of our patients with iliofemoral venous thrombosis had a detection of iliofemoral venous obstruction. Improved lower-extremity swelling was reported in $84 \%$ of the patients following combination therapy involving low-molecularweight heparin (LMWH) and systemic therapy. Conclusion: Patients with an iliofemoral venous thrombosis mainly had iliofemoral venous obstruction by external tumor compression. Combination therapy with LMWH and systemic therapy were mandatory for these patients.

(C) 2016 The Author(s)

Published by S. Karger AG, Basel

\section{Introduction}

Cockett et al. [1] reported that iliofemoral thrombosis is related to iliac vein compression. Unilateral lower limb swelling can be a secondary sign because of the presence of a malignant pelvic tumor [2]. The thrombosis can be of lymphatic or venous origin, or a combination of both as a result of lymphedema in cancer patients. Malignancy recurrence or posttreatment procedures, such as irradiation and/or surgery, can also lead to lymphatic obstruction. Vasogenic edema in malignancy is either related to venous stasis in the vessel lumen or a result of external tumor compression [2,3].

In this case series study, we investigated iliofemoral vein obstruction in cancer patients in correlation with abdominal computed tomography (CT) scans, duplex ultrasound findings, and D-dimer levels. The management of iliofemoral vein obstruction with low-molecularweight heparin (LMWH) was also examined.

\section{Patients and Methods}

\section{Study Population}

Between October 2006 and July 2015, we conducted a retrospective case series study using data collected from patients admitted to the oncology wards of Chang-Gung Memorial Hospital, Taoyuan, Taiwan. A single physician provided most of the data. The physician's field of expertise was urological cancer, and the majority of the patients had urothelial carcinomas. Of these patients, 63 of 829 (8\%) were also diagnosed with iliofemoral venous obstruction.

Diagnosis was based on symptoms, CT findings, and positive duplex ultrasound findings. The criteria for inclusion in the study were: (1) the presence of a unilateral lower-extremity swelling; (2) a CT scan demonstrating a tumor with external compression of the iliac or fem- 


\section{Case Reports in Oncology}

Liao et al.: Iliofemoral Venous Thrombosis Mainly Related to Iliofemoral Venous

Obstruction by External Tumor Compression in Cancer Patients

oral vein, and (3) a duplex ultrasound revealing vein thrombosis or venous flow insufficiency over a femoral vein or saphenous vein.

For the purpose of the correlation between iliofemoral venous obstruction and the presence of iliofemoral venous thrombosis, CT scan rather than venography can evaluate tumor extent and the level of iliac venous obstruction [4, 5]. CT scan in the study traced along from their femoral vein, external iliac vein, common iliac vein and inferior vena cava, and from vascular patency to obliteration by their tumor to detect the compression site. A duplex ultrasound was also done during the presence of a unilateral lower-extremity swelling to detect femoral venous thrombosis or venous flow insufficiency [6-8]. The only exclusion criterion was the presence of bilateral lower-extremity swelling because of the probability of an inferior vena cava lesion. Abnormal venous flow was defined as a condition where venous flow reflux or venous flow insufficiency without the presence of thrombosis occurred [8].

The data regarding each patient that we took into consideration were: (1) the presence of external tumor compression of the iliac or femoral vein, as indicated by CT scan; (2) Ddimer test results; (3) chest plain film findings; (4) complete blood counts; (5) activated partial thromboplastin time (APTT) and prothrombin time (PT) and C-reactive protein (CRP) in selected patients; (6) other thromboembolic complications, and (7) other paraneoplastic syndromes. We applied a cutoff value for the D-dimer test results of $500 \mathrm{ng} / \mathrm{ml}$. Common thromboembolism-associated complications, including symptoms of pulmonary venous obstruction [9], which alter the level of consciousness/change in mental status [10], as well as paraneoplastic pain were observed among these patients. Paraneoplastic pain is defined as breakthrough pain occurring in the absence of an identifiable precipitating cause [11]. Cerebral thromboembolic complications and/or paraneoplastic pain were clinically suspected in most patients because of the difficulty in making a definite diagnosis. Common paraneoplastic syndromes included neoplastic fever (tumor-related fever with good response to the naproxen test) [12], cachexia syndrome (simultaneous weight loss of more than 5\% within 6 months, reduced food intake, and muscle wasting) [13], hypercalcemia (serum calcium level $>11 \mathrm{mg} / \mathrm{dl}$ ), leukemoid reaction (peripheral white cell count $>20,000 / \mu$ l without evidence of infection or leukemia), and prerenal azotemia (defined as a blood urea nitrogen $(\mathrm{BUN}) /$ creatinine ratio $>20)$.

The principle investigation was to assess the correlation between iliofemoral venous obstruction and the presence of iliofemoral venous thrombosis. The variables considered included age, sex, D-dimer value, tumor size, primary tumor, compression location, and cause of compression (lymph nodes or mesenteric tumor).

Treatment was a subcutaneous injection of LMWH (either Fraxiparine, by GlaxoSmithKline, or Enoxaparin, by Sanofi-Aventis), intravenous dexamethasone, and intravenous fluids with/without furosemide when pulmonary vein obstruction syndrome (PVOS) with acute respiratory distress occurred. Further, chemotherapy, targeted therapy, or hormone therapy was conducted depending on the patient's condition. CT scans were obtained from the hospital picture archiving and communication system (PACS). All patients gave verbal informed consent to participate in this study. The protocol of this study was approved by the Institutional Review Board of Chang Gung Memorial Hospital and University (License No. 104-5854B). 


\section{Case Reports in Oncology}

Case Rep Oncol 2016;9:760-771

DOI: $10.1159 / 000452943$

C 2016 The Author(s). Published by S. Karger AG, Basel www.karger.com/cro

Liao et al.: Iliofemoral Venous Thrombosis Mainly Related to Iliofemoral Venous

Obstruction by External Tumor Compression in Cancer Patients

Statistical Methods

Continuous D-dimer level data (presented as mean \pm standard deviation) were gathered, and survival time was calculated from the time of the diagnosis of an iliofemoral venous obstruction to the patient's expiration. Survival curves were determined using the KaplanMeier method. We used the $\chi^{2}$ test in SPSS 23.0.0.2 (IBM) to detect differences between subgroups, and a $p$ value $<0.05$ was considered statistically significant.

\section{Results}

We included 63 consecutive cancer patients (37 men and 26 women; 24-94 years old; median age: 62 ) in this study. The patients' clinical characteristics are shown in Table 1 . The patients had various types of metastatic tumors. Urothelial carcinoma was the most common diagnosis, the urinary tract being the most frequent primary site $(n=46)$, and the bladder $(n=29)$, ureter $(n=8)$, renal pelvis $(n=5)$, prostate $(n=2)$, or penis $(n=2)$ as secondary sites. The second most common primary site was the gastrointestinal tract $(n=9)$, with the colon $(n=3)$, gastric region $(n=3)$, rectum $(n=2)$, or anus $(n=1)$ as secondary sites. The remaining malignant tumors $(n=7)$ were lung $(n=2)$, unknown primary site $(n=2)$, breast $(n=1)$, cervix $(n=1)$, or sarcoma $(n=1)$. Sixteen patients $(25 \%)$ had an Eastern Cooperative Oncology Group (ECOG) performance status of 2 or greater. Common thromboembolic complications occurred in 25 patients (40\%), including consciousness disturbance $(n=16)$, pulmonary venous obstruction $(n=8)$, paraneoplastic pain $(n=3)$, and superficial thrombophlebitis $(n=2)$. Of these 25 patients, 6 were associated with multiple thromboembolic presentations. Of the 16 patients with altered mental status, 2 had evidence of cerebral infarction on magnetic resonance imaging (MRI) and confirmed by angiography. There were 27 patients $(43 \%)$ with paraneoplastic syndromes, 2 with cachexia syndrome and neoplastic fever, and 8 with leukemic like reactions. Of those with paraneoplastic syndromes, 3 had more than 1 class of syndrome.

D-dimer levels and complete blood counts were measured for all patients. The mean Ddimer value was $3,414 \pm 2,369 \mathrm{ng} / \mathrm{ml}(640$ to $>10,000 \mathrm{ng} / \mathrm{ml})$. D-dimer values over 3,000 $\mathrm{ng} / \mathrm{ml}$ were recorded in 36 patients $(57 \%)$. There were 40 patients $(63 \%)$ with hemoglobin levels below $10 \mathrm{~g} / \mathrm{dl}, 23(37 \%)$ with elevated white blood cell counts $(>10,000 / \mu \mathrm{l})$, and 5 $(8 \%)$ with decreased platelet counts $(<100,000 / \mu \mathrm{l})$. APTT and PT were assessed in 22 patients. Of these, $2(3 \%)$ had APTT values $>36 \mathrm{~s}$, and all had PT values $<15 \mathrm{~s}$. Albumin values were $<3.0 \mathrm{~g} / \mathrm{dl}$ in $18(37 \%)$ of the 49 patients. CRP was monitored in 14 patients. The mean CRP value was $86 \pm 74 \mathrm{mg} / \mathrm{l}(55.9-115.1 \mathrm{mg} / \mathrm{l})$.

The correlation of iliofemoral venous obstruction with iliofemoral venous thrombosis is shown in Table 2. Duplex ultrasound was performed in 62 patients, and 1 patient was diagnosed by CT scan only (Fig. 1). Abdominal CT scans were performed in all 63 patients, and revealed external vessel compression caused by a tumor, including femoral vein compression at the inguinal region in 26 patients (41\%), and iliac vein compression in the pelvic region in 37 (59\%). The tumors were characterized as lymph nodes in 33 patients $(53 \%)$ and as mesenteric lesions in 30 patients (47\%), and were $<3 \mathrm{~cm}$ in size in 23 patients $(36 \%)$ and $\geq 3 \mathrm{~cm}$ in $40(64 \%)$. The CT scan of 1 patient also detected a pulmonary embolism (Fig. 2). One patient with bilateral iliofemoral venous thrombosis was diagnosed by duplex ultra- 


\section{Case Reports in Oncology}

Case Rep Oncol 2016;9:760-771

DOI: $10.1159 / 00045294$

\section{(c)}

(C) 2016 The Author(s). Published by S. Karger AG, Basel www.karger.com/cro

Liao et al.: Iliofemoral Venous Thrombosis Mainly Related to Iliofemoral Venous

Obstruction by External Tumor Compression in Cancer Patients

sound. In addition to iliofemoral venous obstruction, 21 (33\%) of the patients also had iliofemoral venous thrombosis, 13 (21\%) had no venous thrombosis but abnormal venous flow (Fig. 3), and the remaining 29 (46\%) had no venous thrombosis and normal venous flow (Table 2). Figure $3 \mathrm{a}, \mathrm{b}$ shows left iliac vein narrowing as a result of external compression caused by the tumor. For 1 of the patients who had no venous thrombosis but abnormal venous flow (according to duplex ultrasound), radionuclide lymphatic venography revealed a lymphatic obstruction at the iliac vein level (Fig. 3c). Patients with femoral vein compression in the inguinal region, D-dimer values $>3,000 \mathrm{ng} / \mathrm{ml}$, cancer of gastrointestinal origin, or invasion of the inguinal lymph nodes had a high risk of iliac venous thrombosis (Table 2). Lower-extremity swelling aggravated by chemotherapy was observed in 2 patients. None of our patients with iliofemoral venous thrombosis had a detection of iliofemoral venous obstruction.

LMWH therapy was administered to 62 of the patients ( 1 patient refused the therapy); 29 were treated with Fraxiparine (3,800 or 5,700 IU daily) and 33 with Enoxaparin (6,000 IU daily). Fifty of the patients treated with LMWH therapy also received additional systemic therapy, including chemotherapy $(n=48)$ and hormone therapy $(n=2)$. The symptom of lower-extremity swelling improved in $42(84 \%)$ of these patients. Seven patients received secondary prevention treatment involving LMWH, and 6 had signs/symptoms of recurrent lower-extremity swelling.

The follow-up period ranged from 1 day to 119 weeks. One patient was lost to follow-up, and 55 were treated and observed until death or by the end of the study (July 2015). Seven patients were still alive by July 2015. The median overall survival time, calculated using the Kaplan-Meier method, was 17 weeks.

\section{Discussion}

Iliofemoral venous obstruction occurred in $8 \%$ of the patients who had been hospitalized at Chang-Gung Memorial Hospital with various types of malignant cancer. All iliofemoral venous obstructions were related to metastatic malignant disease. Iliofemoral venous obstruction is also frequently associated with other thromboembolic complications [13-16], and in addition, a small number of patients exhibited other paraneoplastic syndromes [16]. The situation was similar to Tully's report [17] of a high incidence of vascular thromboembolic events in patients with metastatic or unresectable urothelial cancer treated with platinum chemotherapy.

Virchow described the 3 elements (venous stasis, endothelial injury, and hypercoagulability) that are thought to contribute to venous thromboembolism (VTE) [14]. The mechanism of the prothrombotic state is particularly complex in cancer patients, since cancer cells can activate the hemostatic system via the expression of adhesion molecules, release of inflammatory cytokines, and production of hemostatic factors [15]. However, only one third of the patients with iliofemoral venous obstruction also had iliofemoral venous thrombosis. One fifth of the patients exhibited abnormal venous flow despite having no iliofemoral venous thrombosis. Harris et al. [16] reported that iliofemoral venous obstruction can occur without thrombosis, and Gupta et al. [18] found a case with iliofemoral venous thrombosis caused by external compression with vesical diverticulum. We found that patients with fem- 


\section{Case Reports in Oncology}

oral vein compression in the inguinal region, higher D-dimer levels, cancer of gastrointestinal origin, or lymph node venous compression had a high propensity for iliac venous thrombosis. Most of the patients had inguinal lymph node metastasis. Detection of femoral venous thrombosis was easier with a duplex ultrasound compared to other pelvis locations with tumors close to the femoral vein causing vascular stasis and injury. Gastrointestinal cancer was also characterized by hypercoagulable status than other malignancies. Vascular stasis, vascular injury, and hypercoagulation probably play an important role in thromboembolism, as does iliofemoral venous thrombosis in cancer patients $[14,15]$. All patients in this study showed iliofemoral vein external tumor compression. Cancer of gastrointestinal origin was also characterized by a hypercoagulable status, and lower-extremity swelling aggravated by chemotherapy was observed in 2 patients; hence, cytokines may be involved in the mechanism of the formation of iliofemoral venous obstructions [17].

No iliofemoral venous thrombosis but abnormal venous flow was found in $21 \%$ of the patients [12] in abnormal venous flow states, venous blood refluxes or backward down the veins into an already congested leg [8]. This is related to blockage by tumor external compression in our study.

An iliac lymphatic obstruction was observed in 1 patient. Lymphedema generally has nonpitting characteristics. The location of the edema was related to the iliac lymphatic obstruction in the pelvic area near the lateral abdominal wall. The symptom of unilateral leg swelling, which is related to blockage by tumor, is usually caused by a lymphatic and venous edema. This differs from noncancer etiology; for example, heart disease patients have a higher tendency for bilateral leg edema, with the edema located near the femoral region.

The treatment principle used was according to procoagulant mechanisms. The aim is to relieve external compression and to decrease hypercoagulation. The source of the external compression can be a vascular and/or lymphatic obstruction. Heparin and/or a stent are usually used to treat noncancer-related iliofemoral venous thrombosis, whereas LMWH and combined systemic therapy are used to treat a vascular and/or lymphatic obstruction [1924]. Improvement of lower-extremity swelling was noted in $84 \%$ of the patients, suggesting that the treatment principle followed was correct. Furthermore, we also conducted secondary prevention [20, 22-24].

Our study has several important limitations. First, the data was collected from retrospective case cohort study in a single center mainly from a single physician. Second, the majority of the hospitalized patients had urothelial carcinomas with small case number. None of the patients with iliofemoral venous thrombosis had a detection of iliofemoral venous obstruction and therefore the definite conclusion could not be made. Third, our image studies lack contrast venography and/or radionuclide venography to confirm venous and lymphatic obstruction. Fourth, mental status change related to thromboembolic complication is seldom proven by image study. Fifth, the absence of tissue confirmation of the diagnosis.

In conclusion, cancer patients with an iliofemoral venous thrombosis mainly had iliofemoral venous obstruction by external tumor compression. However, only one third of the patients with this complication also showed signs/symptoms of iliofemoral venous thrombosis. Femoral vein compression in the inguinal region, high D-dimer levels, cancer of gastrointestinal origin, and external vessel compression with lymph node characteristics 


\section{Case Reports in Oncology}

Liao et al.: Iliofemoral Venous Thrombosis Mainly Related to Iliofemoral Venous

Obstruction by External Tumor Compression in Cancer Patients

were strongly related to iliac venous thrombosis. LMWH and systemic therapy were used to treat these patients. Continuous maintenance of LMWH and systemic therapy are highly recommended.

\section{Acknowledgment}

The authors appreciated our oncology nurse staff for providing the best care for these patients.

\section{Statement of Ethics}

The authors declared that an institutional review board/ethics committee determined that informed consent was not required. The study was approved by the investigator's institutional review board/ethics committee.

\section{Disclosure Statement}

The authors declared that they have no conflicts of interests regarding the publication of this paper.

\section{References}

1 Cockett FB, Thomas ML, Negus D: Iliac vein compression: its relation to iliofemoral thrombosis and the post-thrombotic syndrome. Br Med J 1967;2:14-19.

$\checkmark 2$ Abu-Rustum NR, Alektiar K, Iasonos A, Lev G, Sonoda Y, Aghajanian C, Chi DS, Barakat RR: The incidence of symptomatic lower-extremity lymphedema following treatment of uterine corpus malignancies: a 12-year experience at Memorial Sloan-Kettering Cancer Center. Gynecol Oncol 2006;103:714-718.

-3 Loud PA, Klippenstein DL: Lower extremity deep venous thrombosis in cancer patients: correlation of presenting symptoms with venous sonographic findings. J Ultrasound Med 1998;17:693-696.

4 Xiao L, Tong JJ, She J: Endoluminal treatment for venous vascular complications of malignant tumors. Exp Ther Med 2012;4:323-328.

-5 Oguzkurt L, Tercan F, Pourbagher MA, Kizilkilic 0, Turkoz R, Boyvat F: Computed tomography findings in 10 cases of iliac vein compression (May-Thurner) syndrome. Eur J Radiol 2005;55:421-425.

6 Gornik HL, Sharma AM: Duplex ultrasound in the diagnosis of lower-extremity deep venous thrombosis. Circulation 2014;129:917-921.

7 Kayılığlu SI , Köksoy C, Alaçayır İ: Diagnostic value of the femoral vein flow pattern for the detection of an iliocaval venous obstruction. J Vasc Surg Venous Lymphat Disord 2016;4:2-8.

-8 Metzger PB, Rossi FH, Kambara AM, NM Izukawa, Saleh MH, Pinto IM, Amorim JE, Thorpe PE: Criteria for detecting significant chronic iliac venous obstructions with duplex ultrasound. J Vasc Surg Venous Lymphat Disord 2016;4:18-27.

-9 Liaw CC, Chang H, Yang TS, Wen MS: Pulmonary venous obstruction in cancer patients. J Oncol 2015;2015:210916.

10 Schwarzbach CJ, Schaefer A, Ebert A, Held V, Bolognese M, Kablau M, Fatar M: Stroke and cancer: the importance of cancer-associated hypercoagulation as a possible stroke etiology. Stroke 2012;43:30293034. 


\section{Case Reports in Oncology}

Liao et al.: Iliofemoral Venous Thrombosis Mainly Related to Iliofemoral Venous

Obstruction by External Tumor Compression in Cancer Patients

11 Jost L, Roila F: Management of cancer pain: ESMO clinical recommendations. Ann Oncol 2010;2(suppl 5):257-260.

12 Chang JC, Gross HM: Neoplastic fever responds to the treatment of an adequate dose of naproxen. J Clin Oncol 1985;3:552-558.

13 Fearon KC, Voss AC, Hustead DS; Cancer Cachexia Study Group: Definition of cancer cachexia: effect of weight loss, reduced food intake, and systemic inflammation on functional status and prognosis. Am J Clin Nutr 2006;83:1345-1350.

14 Bagot CN, Arya R: Virchow and his triad: a question of attribution. Br J Haematol 2008;143:180-190.

15 Falang M, Marchetti A, Vignoli A: Coagulation and cancer: biological and clinical aspects. Thromb Haemost 2013;11:223-233.

16 Harris RW, Andros G, Dulawa LB, Oblath RW, Horowitz R: Iliofemoral venous obstruction without thrombosis. J Vasc Surg 1987;6:594-599.

17 Tully CM, Apolo AB, Zabor EC, Regazzi AM, Ostrovnaya I, Furberg HF, Rosenberg JE, Bajorin DF: The high incidence of vascular thromboembolic events in patients with metastatic or unresectable urothelial cancer treated with platinum chemotherapy. Cancer 2016;122:712-721.

18 Gupta V, Shaik I, Abbas J, Nazzal M: Iliofemoral venous thrombosis from external compression by a vesical diverticulum. J Vasc Surg 2010;52:1671-1673.

19 Streiff MB, Holmstrom B, Ashrani A, Bockenstedt PL, Chesney C, Eby C, Fanikos J, Fenninger RB, Fogerty AE, Gao S, Goldhaber SZ, Hendrie P, Kuderer N, Lee A, Lee JT, Lovrincevic M, Millenson MM, Neff AT, Ortel TL, Paschal R, Shattil S, Siddiqi T, Smock J, Soff G, Wang TF, Yee GC, Zakarija A, McMillian N, Engh AM: Cancer-associated venous thromboembolic disease, Version 1.2015. J Natl Compr Canc Netw 2015;13:1079-1095.

20 Francis CW, Kessler CM, Goldhaber SZ, Kovacs MJ, Monreal M, Huisman MV, Bergqvist D, Turpie AG, Ortel TL, Spyropoulos AC, Pabinger I, Kakkar AK: Treatment of venous thromboembolism in cancer patients with dalteparin for up to 12 months: the DALTECAN Study. J Thromb Haemost 2015;3:10281035.

21 Comerota AJ, Gravett MH: Iliofemoral venous thrombosis. J Vasc Surg 2007;46:1065-1076.

-22 Mandalà M, Falanga A, Roila F: Management of venous thromboembolism (VTE) in cancer patients: ESMO Clinical Practice Guidelines. Ann Oncol 2011;22(suppl 6):vi85-vi92.

23 Watson HG, Keeling DM, Laffan M, Tait RC, Makris M: British Committee for Standards in Haematology. Guideline on aspects of cancer-related venous thrombosis. Br J Haematol 2015;17:640-648.

24 Bock RL: Cancer-associated thrombosis: focus on extended therapy with dalteparin. J Support Oncol 2006;4:115-120.

T.-Y.L., H.-C.H., M.-S.W., Y.-H.J., and Y.-H.H. contributed equally to this work. 


\section{Case Reports in Oncology}
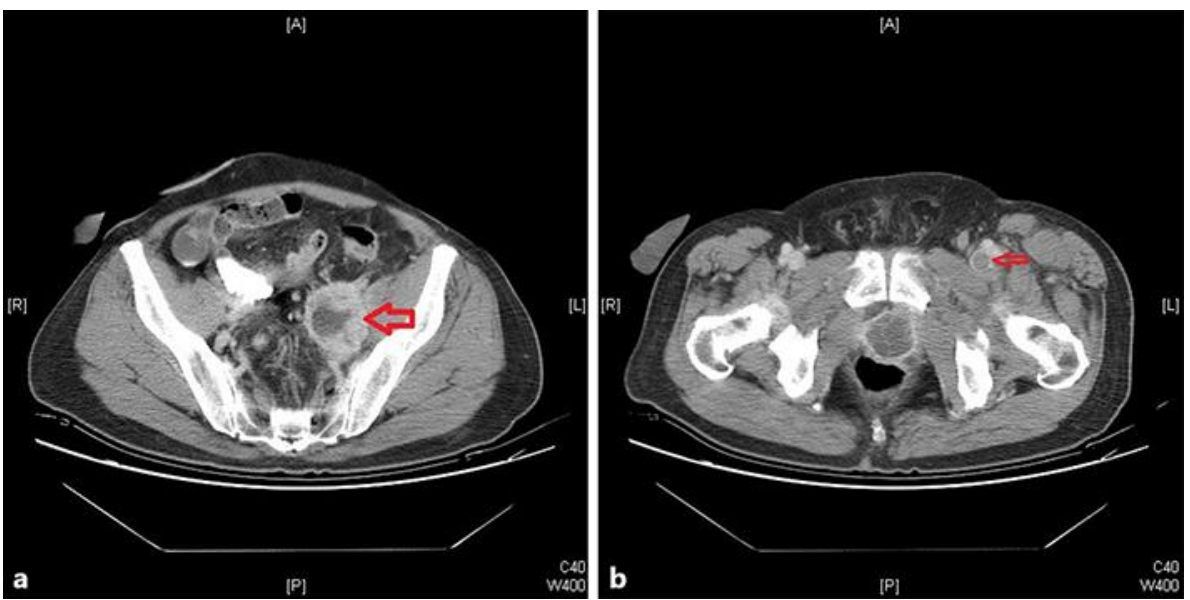

Fig. 1. A 61-year-old man with recurrent urinary bladder urothelial carcinoma presented with left lowerextremity swelling. The CT scan showed external compression of the left iliac vein (a). Left iliac lymph node: femoral vein thrombosis was located in the left inguinal region (b).
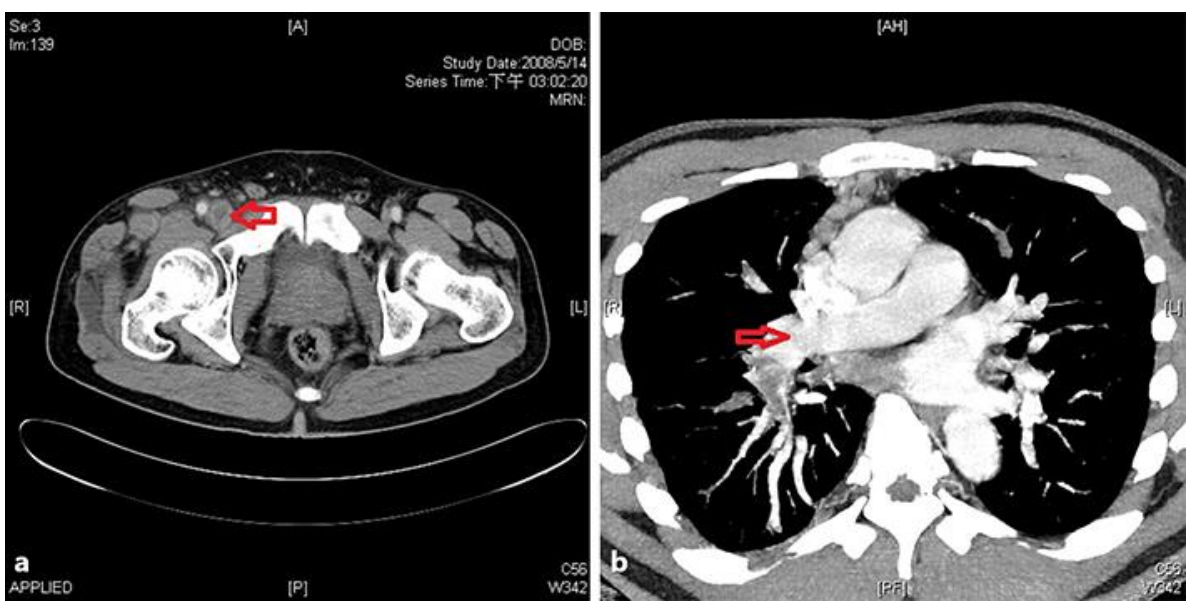

Fig. 2. A 41-year-old man with lung adenocarcinoma presented with right lower-extremity swelling. The CT scan showed femoral vein thrombosis located in the right inguinal region due to external compression by right inguinal lymph node (a). Right pulmonary embolism was also present (b). 


\section{Case Reports in Oncology}
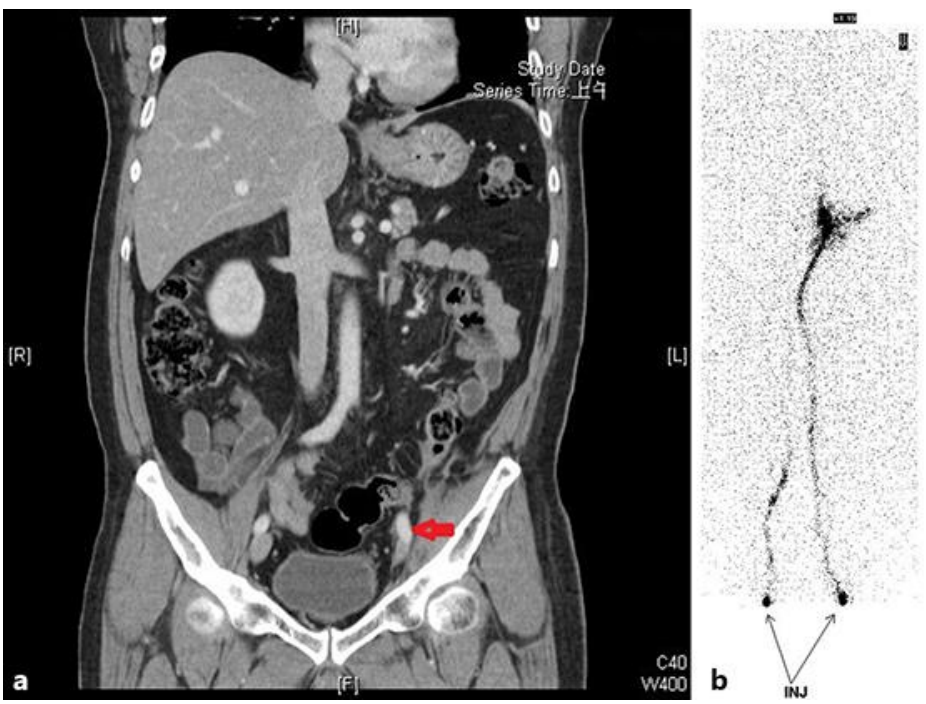

Fig. 3. A 51-year-old man had left ureteral urothelial carcinoma with mesenteric seeding. He presented with left lower-extremity swelling. The CT scan revealed tumor compression of the left iliac vein with narrowing (coronary view) (a). Radionuclide venography showed a left iliac vein obstruction (b). 


\section{Case Reports in Oncology}

Liao et al.: Iliofemoral Venous Thrombosis Mainly Related to Iliofemoral Venous

Obstruction by External Tumor Compression in Cancer Patients

Table 1. Characteristics of 63 cancer patients with iliofemoral venous obstruction

\begin{tabular}{ll} 
Characteristics & No. of patients (\%) \\
\hline All patients & 63 \\
Median age, years (range) & $62(24-94)$ \\
Sex & $37 / 36$ \\
$\quad$ Male/female & \\
Primary sites, $n$ & $46(73)$ \\
$\quad$ Urinary tract & $9(14)$ \\
$\quad$ Gastrointestinal tract & $8(13)$ \\
$\quad$ Others & \\
Disease extent, $n$ & $63(100)$ \\
$\quad$ Metastatic disease & $0(0)$ \\
$\quad$ Locoregionally advanced disease & \\
Performance status, $n$ & $47(75)$ \\
$\quad$ 0-1 & $16(25)$ \\
$\quad \geq 2$ & \\
Associated with other thromboembolic & \\
complications, $n$ & \\
$\quad$ Yes & $25(40)$ \\
$\quad$ No & $38(60)$ \\
Associated with other paraneoplastic & \\
syndrome, $n$ & \\
$\quad$ Yes &
\end{tabular}




\section{Case Reports in Oncology}

Liao et al.: Iliofemoral Venous Thrombosis Mainly Related to Iliofemoral Venous

Obstruction by External Tumor Compression in Cancer Patients

Table 2. Correlation of iliofemoral venous thrombosis with iliofemoral venous obstruction

\begin{tabular}{lcc}
\hline $\begin{array}{l}\text { Presence of iliofemoral venous } \\
\text { thrombosis }\end{array}$ & $\begin{array}{l}\text { No. of } \\
\text { patients (\%) }\end{array}$ & $p$ value \\
\hline $\begin{array}{l}\text { Total, } n / \text { total } n \\
\text { Age, } n / \text { total } n\end{array}$ & $21 / 63(33)$ & \\
$\quad \geq 50$ years & & 0.285 \\
$\quad<50$ years & $7 / 22(32)$ & \\
Sex, $n /$ total $n$ (\%) & $14 / 41(55)$ & \\
$\quad$ Male & & 0.366 \\
$\quad$ Female & $7 / 26(28)$ & \\
D-dimer, $n /$ total $n$ & $14 / 37(38)$ & \\
$\quad<3,000$ ng/ml level & & \\
$\quad \geq 3,000$ ng/ml level & $3 / 27(11)$ & \\
\hline Primary cancer, $n /$ total $n$ & $18 / 36(48)$ & \\
$\quad$ GI cancer & & \\
$\quad$ Non-GI cancer & $7 / 9(78)$ & \\
Anatomy location, $n /$ total $n$ & $14 / 54(26)$ & \\
$\quad$ Inguinal area & & \\
$\quad$ Iliac area & $14 / 26(53)$ & \\
Tumor size, $n /$ total $n$ & $7 / 37(19)$ & \\
$\quad \geq 3$ cm & & \\
$\quad<3$ cm & $5 / 40(13)$ & \\
Tumor characteristic, $n /$ total $n$ & $16 / 23(70)$ & \\
$\quad$ Lymph nodes & & \\
$\quad$ Mesentery & $14 / 33(42)$ & \\
\hline
\end{tabular}

One patient was found to have iliofemoral venous thrombosis via CT scan, instead of duplex ultrasound (not done). One patient was diagnosed with bilateral iliofemoral venous thrombosis by duplex ultrasound. 